\title{
Predicting Intestinal Viability After Acute Mesenteric Ischemia by Photoacoustic Imaging: An Experimental Study in Rats
}

\author{
Takumi Sugiura \\ Kanazawa University \\ Kenichiro Okumura ( $\Delta$ okumurak@staff.kanazawa-u.ac.jp ) \\ Kanazawa University \\ Junichi Matsumoto \\ Kanazawa University \\ Maki Sakaguchi \\ Kanazawa University \\ Takahiro Komori \\ Kanazawa University \\ Takahiro Ogi \\ Kanazawa University \\ Dai Inoue \\ Kanazawa University \\ Wataru Koda \\ Kanazawa University \\ Satoshi Kobayashi \\ Kanazawa University \\ Toshifumi Gabata \\ Kanazawa University
}

\section{Research Article}

Keywords: photoacoustic imaging, mesenteric and marginal vessels, reperfusion, pathological severity

Posted Date: June 10th, 2021

DOI: https://doi.org/10.21203/rs.3.rs-565180/v1

License: (1) (1) This work is licensed under a Creative Commons Attribution 4.0 International License.

Read Full License 


\section{Abstract}

Our purpose was to assess the ability of photoacoustic imaging (PAI) to objectively predict ischemic intestinal pathological damage during surgery for acute mesenteric ischemia (AMI).

PAl uses laser light to detect relative amounts of oxygenated and deoxygenated hemoglobin in intestinal tissue. In 18 rats, AMI was induced by clamping the mesenteric and marginal vessels of the ileum for 0 minutes in the sham group $(n=6), 30$ minutes in the mild group $(n=6)$, and 180 minutes in the severe group $(n=6)$. After 60 minutes of reperfusion, the damage of the intestine was evaluated pathologically. Oxygenation of the intestine was monitored throughout the procedure in real time by the PAI system and compared between the mild and severe groups.

All rats showed irreversible (i.e. transmucosal or transmural infarction) damage in the severe group. After reperfusion, the oxygenation in the mild group recovered immediately and was significantly higher than in the severe group at $1,5,10,30$, and 60 minutes $(P=.007,014,016,008$, and 003 , respectively). Oxygenation showed a significant strong negative correlation with the pathological severity.

In conclusion, PAl could objectively predict irreversible ischemic damage immediately after reperfusion, which potentially prevents inadequate surgery.

\section{Introduction}

During surgery for acute mesenteric ischemia (AMI), since there is no reliable objective marker of intestinal viability to determine the resection margin, it is assessed subjectively by the surgeon. However, this requires a high level of clinical experience ${ }^{1-4}$.

In recent years, photoacoustic imaging (PAI), also known as optoacoustic imaging, has been demonstrating its usefulness in a variety of fields, including clinical practice ${ }^{5-13}$. It is an emerging realtime in vivo imaging modality that combines optical imaging contrast with ultrasonic spatial resolution. PAl uses the near-infrared spectrum, which has deeper penetration and visualizes deeper tissue in vivo in real time. Light energy is selectively absorbed by endogenous and exogenous chromophores in tissue causing rapid thermoelastic expansion, which generates broadband acoustic waves that can be detected by ultrasound transducers ${ }^{14}$. Differing absorption spectra of oxygenated hemoglobin $\left(\mathrm{HbO}_{2}\right)$ and deoxyhemoglobin $(\mathrm{Hb})$ with dual-wavelength PAI allow quantification of the blood oxygen level. The usefulness of oxygenation mapping in organ ischemia has also been reported ${ }^{15,16}$.

With PAl, oxygenation of ischemic intestine after reperfusion can be evaluated noninvasively during surgery. The purpose of this study was to evaluate the usefulness of oxygenation mapping by PAI to objectively and quantitatively predict ischemic intestinal pathological damage during surgery for acute mesenteric ischemia (AMI).

\section{Results}




\section{Laboratory tests}

Results of laboratory tests are shown in Fig. 1(A). Local capillary lactate was significantly higher in the severe group $(14.2 \pm 8.6 \mathrm{mmol} / \mathrm{L})$ than in the mild group and the sham group $(4.1 \pm 3.1$ and $1.0 \pm 0.1$ $\mathrm{mmol} / \mathrm{L}, P=.01$ and .002 , respectively). There was no significant difference between the mild group and the sham group $(P=.58)$. Systemic lactate was significantly higher in the severe group and in the mild group $(0.6 \pm 0.1$ and $0.7 \pm 0.2 \mathrm{mmol} / \mathrm{L})$ than in the sham group $(0.4 \pm 0.1 \mathrm{mmol} / \mathrm{L}, P=.03,001$, respectively), but there was no significant difference between the severe group and the mild group ( $P$ $=.27$ ). $\mathrm{LDH}$ (severe, $431.3 \pm 131.0$; mild, $188.7 \pm 34.9$; sham, $139.7 \pm 18.5 \mathrm{U} / \mathrm{L}$; respectively) and CK (severe, $406.2 \pm 144.5$; mild, $143.3 \pm 11.8$; sham, $125.8 \pm 23.1 \mathrm{U} / \mathrm{L}$; respectively) were significantly higher in the severe group than in the others (all $P<.001$, for details, see Fig. 1(A)), but there was no significant difference between the mild and the sham groups $(P=.54$ and .93$)$. AST did not show a significant difference between any two groups (severe, $73.5 \pm 20.5$; mild, $64.3 \pm 8.9$; sham, $63.0 \pm 5.9 \mathrm{U} / \mathrm{L}$; respectively. $P=.36$ ).

\section{Time course of oxygenation in AMI}

Before clamping, average oxygenation was greater than $70 \%$. All rats showed a rapid decrease of oxygenation after clamping, and then reached a plateau $(<30 \%)$ within five minutes. The time course of the oxygenation after clamping is shown in Fig. 1(B).

The appearance of ischemic intestine was dark, and it tended to be darker in the severe group, but it was not always distinguishable between the two ischemic groups (Fig. 2).

Figure 3(A), Fig. 3(B), and Table 1 show the time course of oxygenation in two groups after reperfusion. Before removing the vascular clamps, there was no significant difference between the two groups. Once the vascular clamps were removed, oxygenation recovered to the baseline level (about 65-75\%) immediately in the mild group. On the other hand, oxygenation recovered slightly but remained lower in the severe group. At 1, 5, 10,30, and 60 minutes after the removal of vascular clamps, oxygenation was significantly lower in the severe group than in the mild group. 
Table 1

Oxygenation after reperfusion in the mild group and the severe group

\begin{tabular}{|llll|}
\hline Time (min) & Oxygenation in the mild group & Oxygenation in the severe group & $P$ value \\
\hline 0 & $17.6(2.2)$ & $24.9(2.6)$ & .30 \\
\hline 1 & $65.3(3.2)$ & $34.2(5.3)$ & .006 \\
\hline 5 & $68.8(1.9)$ & $33.8(5.8)$ & .007 \\
\hline 10 & $69.6(3.0)$ & $29.4(4.3)$ & $<.001$ \\
\hline 30 & $65.7(1.6)$ & $33.5(4.3)$ & .002 \\
\hline 60 & $66.8(3.1)$ & $33.8(4.6)$ & .001 \\
\hline
\end{tabular}

Note - After reperfusion, the time course of oxygenation was clearly different between the mild group and the severe group. Oxygenation was significantly higher in the mild group than in the severe group at 1, 5, 10, 30, and 60 min. All values are shown as means (standard error).

\section{Pathological findings}

In the severe group, all tissue samples showed irreversible damage, and the average score was $7.2 \pm 0.4$, significantly higher than in the other groups (both $P<.001$ ). In the mild group, the average score was 1.2 \pm 1.8 , and in the sham group, the average score was 0 .

\section{Correlation analysis between oxygenation and the pathological score}

Correlation analysis between oxygenation and the pathological score was performed (Fig. 3(C), Table 2). Table 2 shows Spearman's correlation coefficient $\left(\mathrm{r}_{\mathrm{s}}\right.$-value) along with the corresponding $P$-values for the correlation between oxygenation and the pathological score, showing a significant strong negative correlation at each time point after reperfusion. Correlation analysis between local capillary lactate and the pathological score was also performed, but it did not show a significant correlation $(P=0.06)$. 
Table 2

Correlation between oxygenation and the pathological score

\begin{tabular}{|lll|}
\hline Time (min) & $P$ value & $\mathrm{r}_{\mathbf{s}}$ value \\
\hline $\mathbf{0}$ & .065 & .554 \\
$\mathbf{1}$ & .007 & -0.754 \\
$\mathbf{5}$ & .014 & -0.706 \\
\hline 10 & .016 & -0.695 \\
\hline 30 & .008 & -0.747 \\
\hline 60 & .003 & -0.806 \\
\hline
\end{tabular}

Note - The $P$ value and $\mathrm{r}_{\mathrm{s}}$ value between oxygenation and the pathological score are shown. There was a significant, strong negative correlation between oxygenation and the pathological score at 1, 5, 10, 30, and $60 \mathrm{~min}$.

\section{Discussion}

During AMI surgery, intestinal viability is subjectively assessed by surgeons and is highly dependent on their experience. Karliczek et al. evaluated surgeons' predictive accuracy for anastomotic leakage in gastrointestinal surgery, but the predictive value was low in a prospective, clinical study. Incorrect evaluation may result in bowel stricture, obstruction, necrosis, anastomotic leakage, and short bowel syndrome ${ }^{2,17}$. Thus, the usefulness of oxygenation mapping by PAI as an objective, quantitative, in vivo method to assess intestinal viability in AMI was evaluated. In the present study, there was a significant difference in the time course of oxygenation immediately after reperfusion between the two ischemic groups with different prognoses. In addition, oxygenation in the intestine at each time point showed a significant strong negative correlation between the pathological scores, even at 1 minute after reperfusion. To the best of our knowledge, this is the first report to evaluate intestinal viability in AMI with oxygenation mapping by PAl.

\section{Oximetry with Photoacoustic imaging}

Singh et al. showed that the serosal oxygenation measurements were not sensitive to ischemia ${ }^{18}$. It has been estimated that blood flow in the mucosal and submucosal layers accounts for $70 \%$ of total blood flow in intestinal tissues; therefore, microcirculation should be evaluated in the mucosal and submucosal layers ${ }^{19}$. There are various methods to evaluate intestinal viability during surgery, and most of them reflect oxygenation and perfusion from the serosal surface, but none of them has become a clinical standard due to various deficiencies ${ }^{2,20}$. In recent years, PAl has been demonstrating its clinical usefulness, which can image deeper tissue and can be performed at the bedside in real time ${ }^{5,8,10,11}$. It is 
worth mentioning that PAI can be performed during surgery as well as ultrasound. With this method, oxygenation of the layers including the mucosal layer can be evaluated noninvasively. In the present study with the preclinical PAI system, layers including the mucosal and submucosal layers were evaluated.

\section{Sequential real-time evaluation of oxygenation}

In the present study, before inducing AMI, average oxygenation of normal ileum was between $70 \%$ and $80 \%$, which was compatible with the previous report using visual light spectroscopy ${ }^{17}$. In the early stage of $\mathrm{AMI}$, oxygenation reached a plateau within five minutes. From the present result, intestinal viability cannot be predicted by the absolute oxygenation value itself before reperfusion. This finding is clinically acceptable. In clinical practice, decreased staining of the intestinal wall on contrast-enhanced computed tomography is a key finding for diagnosing AMI, but an intestinal segment with decreased staining is not always irreversibly damaged after reperfusion.

The decrease in oxygenation can be caused either by a decrease in arterial blood with rich oxygenation or by possible pooling of venous blood. The significantly low oxygenation after reperfusion in the severe AMl group may due to vasospasm, thrombosis, and congestion in damaged intestinal tissue ${ }^{21}$.

The present study has limitations. First, in strangulated bowel obstruction in clinical practice, venous flow is compromised prior to arterial flow. In the present model, both arterial and venous flow were obstructed simultaneously. Second, the reason why oxygenation decreased was not specifically clarified. In addition, the regions of interest in intestinal tissue on PAl could not be maintained continuously in a particular (mucosal and submucosal) layer under respiratory motion, due to the small size of rats; thus, oxygenation was evaluated using regions of interest including all layers.

\section{Conclusion}

Real-time PAI could objectively and quantitatively predict irreversible ischemic tissue damage immediately after reperfusion in AMI, and it can be performed at the bedside and potentially prevent inadequate surgery.

\section{Material Methods}

\section{Animal models}

Eighteen male Sprague-Dawley rats provided by Charles River Laboratories Japan (Yokohama, Japan) were studied. All animal experiments were performed in accordance with the guidelines of our institution and approved by our animal research committee (study protocol ID: AP194060). Rats were maintained in a specific pathogen-free facility. All rats weighed approximately $600 \mathrm{~g}$ and had ad libitum access to food and water. AMI was established by the procedure described below.

\section{Rat model of AMI}


After 24 hours of fasting, the abdomen was shaved. A 3-cm midline incision was made, and a 15-cm length of ileum from the ileocecal valve was exposed. Blood samples were obtained from the femoral vein to evaluate systemic lactate, lactate dehydrogenase (LDH), aspartate transaminase (AST), and creatine phosphokinase (CK) levels. Subsequently, local capillary lactate was evaluated by puncturing the intestine. The rats were randomly assigned into three groups in advance. The first group was the sham group $(n=6)$, which was designed to assess the effect of anesthesia and the invasiveness of the operation to standardize the study in terms of ischemia. The second group was the mild AMI group ( $\mathrm{n}=$ 6), in which mesenteric arteries and veins and marginal arteries and veins were clamped with vascular clamps, and mesenteric ischemia was then induced in $15 \mathrm{~cm}$ of ileum from the ileocecal valve (strangulated bowel obstruction model; Fig. 4); after 30 minutes of ischemia, the clamps were removed, and blood samples were obtained, and local capillary lactate was again evaluated; after 60 minutes of reperfusion, a tissue sample was obtained for pathological evaluation. The third group was the severe AMI group $(n=6)$, which was the same as the mild AMI group, but with 180 minutes of ischemia. During $\mathrm{AMI}$ and reperfusion, oxygenation of ischemic intestine was monitored by a commercially available PAI system Vevo LAZR /Vevo 2100 (FUJIFILM VisualSonics Inc., Toronto, Canada, Fig. 5(A)).

Induction of anesthesia was performed with $5 \%$ isoflurane, and it was then maintained by intraperitoneal injection of a mixture of medetomidine hydrochloride $(0.15 \mathrm{mg} / \mathrm{kg})$, midazolam $(2.0 \mathrm{mg} / \mathrm{kg})$, and butorphanol tartrate $(2.5 \mathrm{mg} / \mathrm{kg})$. Vital signs (heart rate, respiratory rate, peripheral oxygen saturation) were continuously monitored by MouseOx PLUS (Starr Life Sciences Co., PA, USA) to exclude hypoventilation. The neck of each rat was also shaved, and a clip sensor was affixed. During the procedure, the rats were kept on a heating pad to avoid hypothermia.

\section{Ultrasound and PAI}

PAI was performed using a Vevo LAZR/2100 imaging system with an LZ-550 linear-array transducer (256 elements, center frequency $40 \mathrm{MHz}$, bandwidth $27 \mathrm{MHz}$ ) used to acquire all images. The tunable laser supplied 10-20 $\mathrm{mJ}$ per pulse over the 680-970 $\mathrm{nm}$ wavelength range, with a pulse repetition frequency of $10 \mathrm{~Hz}$. Once initialized, the system was switched to "Oxy-Hemo" mode to obtain parametric maps of the oxygenation using the following parameters: depth $6 \mathrm{~mm}$; width $14.08 \mathrm{~mm}$; and wavelength 750 and $850 \mathrm{~nm}$. The oxygenation mapping was shown in parallel with B-mode images (Fig. 5(B)). Before the removal of vascular clamps, and 1, 5, 10,30 and 60 minutes after removal, five regions of interest were placed on layers including the mucosal layer, submucosal layer, muscular layer, and subserosal layer, and oxygenation was measured for 10 seconds and averaged. Oxygenation of the mild and severe groups was compared.

\section{Pathological findings}

At the end of each experiment, a tissue sample was obtained for histopathological study. Tissues were stained with hematoxylin and eosin and evaluated by a pathologist blinded to group assignment. The sections from the intestine were classified according to the degree of tissue injury in accordance with Chiu's score classification including modifications proposed by Park ${ }^{22,23}$. This Park/Chiu's classification 
of small intestinal injury consists of values from 0 to 8 , which are shown in Table 3. Samples of pathological findings are shown in Fig. 6. Scores of 7 and 8 were considered to indicate irreversible ischemic damage.

Table 3

Scoring system for assessment of ischemic damage

\begin{tabular}{|ll|}
\hline Grade & Description \\
\hline $\mathbf{0}$ & Normal mucosa \\
\hline $\mathbf{1}$ & Subepithelial space at villus tips \\
\hline $\mathbf{2}$ & Extension of subepithelial space with moderate lifting \\
\hline $\mathbf{3}$ & Massive lifting down sides of villi, some denuded tips \\
\hline $\mathbf{4}$ & Denuded villi, dilated capillaries \\
\hline $\mathbf{5}$ & Disintegration of lamina propria \\
\hline $\mathbf{6}$ & Crypt layer injury \\
\hline $\mathbf{7}$ & Transmucosal infarction \\
\hline $\mathbf{8}$ & Transmural infarction \\
\hline
\end{tabular}

Note - A commonly used pathological classification system for ischemic mucosal lesions proposed by Chiu et al [22], including modifications proposed by Park et al [23].

\section{Statistical Analysis}

All results are expressed as means \pm standard deviation unless otherwise noted. Statistical analysis and graphical displays of data were performed using GraphPad software (version 8.3.0 for Mac; GraphPad Software, San Diego, CA, USA). One-way analysis of variance (ANOVA) with Tukey's multiple comparison test and two-way ANOVA with Sidak multiple comparisons were performed to evaluate the values of laboratory tests and oxygenation at different time points. Prior to the ANOVA test, Shapiro-Wilk test was performed and $P$-value $>.05$ was considered normally distributed. Correlation was evaluated by linear regression and Spearman's correlation coefficient $\left(r_{s}\right.$-value). A $P$-value $<.05$ was considered significant.

\section{Abbreviations}

$\mathrm{AMI}=$ Acute mesenteric ischemia

PAl = Photoacoustic imaging

\section{Declarations}

Conflict of interest: The authors declare that they have no conflict of interest. 
Funding: This research was supported by JSPS KAKENHI grant number $18 \mathrm{~K} 15543$ to T.K.

\section{Acknowledgments}

This work was supported by JSPS KAKENHI grant number 18K15543.

The authors would like to thank the staff of the Institute of Experimental Animals at ${ }^{* \star \star \star(b l i n d e d)}$ Universi-ty for their technical assistance in performing our studies.

\section{References}

1. Sheridan, W. G., Lowndes, R. H., Williams, G. T. \& Young, H. L. Determination of a critical level of tissue oxygenation in acute intestinal ischaemia. Gut 33, 762-766, doi:10.1136/gut.33.6.762 (1992).

2. Urbanavicius, L., Pattyn, P., de Putte, D. V. \& Venskutonis, D. How to assess intestinal viability during surgery: A review of techniques. World J Gastrointest Surg 3, 59-69, doi:10.4240/wjgs.v3.i5.59 (2011).

3. Ghodasara, N., Liddell, R., Fishman, E. K. \& Johnson, P. T. High-Value Multidetector CT Angiography of the Superior Mesenteric Artery: What Emergency Medicine Physicians and Interventional Radiologists Need to Know. Radiographics 39, 559-577, doi:10.1148/rg.2019180131 (2019).

4. Kanasaki, S. et al. Acute Mesenteric Ischemia: Multidetector CT Findings and Endovascular Management. Radiographics 38, 945-961, doi:10.1148/rg.2018170163 (2018).

5. Neuschler, E. I. et al. A Pivotal Study of Optoacoustic Imaging to Diagnose Benign and Malignant Breast Masses: A New Evaluation Tool for Radiologists. Radiology 287, 398-412, doi:10.1148/radiol.2017172228 (2018).

6. Attia, A. B. E. et al. A review of clinical photoacoustic imaging: Current and future trends. Photoacoustics 16, 100144, doi:10.1016/j.pacs.2019.100144 (2019).

7. Steinberg, I. et al. Photoacoustic clinical imaging. Photoacoustics 14, 77-98, doi:10.1016/j.pacs.2019.05.001 (2019).

8. Suzuki, Y. et al. Subcutaneous Lymphatic Vessels in the Lower Extremities: Comparison between Photoacoustic Lymphangiography and Near-Infrared Fluorescence Lymphangiography. Radiology, 191710, doi:10.1148/radiol.2020191710 (2020).

9. Valluru, K. S., Wilson, K. E. \& Willmann, J. K. Photoacoustic Imaging in Oncology: Translational Preclinical and Early Clinical Experience. Radiology 280, 332-349, doi:10.1148/radiol.16151414 (2016).

10. Nandy, S. et al. Evaluation of Ovarian Cancer: Initial Application of Coregistered Photoacoustic Tomography and US. Radiology 289, 740-747, doi:10.1148/radiol.2018180666 (2018).

11. Menezes, G. L. G. et al. Downgrading of Breast Masses Suspicious for Cancer by Using Optoacoustic Breast Imaging. Radiology 288, 355-365, doi:10.1148/radiol.2018170500 (2018). 
12. Okumura, K. et al. Photoacoustic imaging of tumour vascular permeability with indocyanine green in a mouse model. Eur Radiol Exp 2, 5, doi:10.1186/s41747-018-0036-7 (2018).

13. Karlas, A. et al. Cardiovascular optoacoustics: From mice to men - A review. Photoacoustics 14, 1930, doi:10.1016/j.pacs.2019.03.001 (2019).

14. Needles, A. et al. Development and initial application of a fully integrated photoacoustic microultrasound system. IEEE Trans Ultrason Ferroelectr Freq Control 60, 888-897, doi:10.1109/tuffc.2013.2646 (2013).

15. Mukaddim, R. A., Rodgers, A., Hacker, T. A., Heinmiller, A. \& Varghese, T. Real-Time in Vivo Photoacoustic Imaging in the Assessment of Myocardial Dynamics in Murine Model of Myocardial Ischemia. Ultrasound Med Biol 44, 2155-2164, doi:10.1016/j.ultrasmedbio.2018.05.021 (2018).

16. Okumura, K. et al. Evaluation of renal oxygen saturation using photoacoustic imaging for the early prediction of chronic renal function in a model of ischemia-induced acute kidney injury. Plos one 13, e0206461, doi:10.1371/journal.pone.0206461 (2018).

17. Karliczek, A. et al. Intraoperative assessment of microperfusion with visible light spectroscopy for prediction of anastomotic leakage in colorectal anastomoses. Colorectal Dis 12, 1018-1025, doi:10.1111/j.1463-1318.2009.01944.x (2010).

18. Singh, D. B., Bain, I. \& Harrison, D. K. Use of visible spectrophotometry to assess tissue oxygenation in the colostomy stoma. Adv Exp Med Biol 662, 273-278, doi:10.1007/978-1-4419-1241-1_39 (2010).

19. Gore, R. W. \& Bohlen, H. G. Microvascular pressures in rat intestinal muscle and mucosal villi. Am J Physiol 233, H685-693, doi:10.1152/ajpheart.1977.233.6.H685 (1977).

20. Horgan, P. G. \& Gorey, T. F. Operative assessment of intestinal viability. Surg Clin North Am 72, 143155, doi:10.1016/s0039-6109(16)45632-x (1992).

21. Granger, D. N., Seifert, H. \& Senchenkova, E. in PanVascular Medicine (ed Peter Lanzer) 3535-3553 (Springer Berlin Heidelberg, 2015).

22. Chiu, C. J., McArdle, A. H., Brown, R., Scott, H. J. \& Gurd, F. N. Intestinal mucosal lesion in low-flow states. I. A morphological, hemodynamic, and metabolic reappraisal. Arch Surg 101, 478-483, doi:10.1001/archsurg.1970.01340280030009 (1970).

23. Park, P. O., Haglund, U., Bulkley, G. B. \& Fält, K. The sequence of development of intestinal tissue injury after strangulation ischemia and reperfusion. Surgery 107, 574-580 (1990).

\section{Figures}


A)
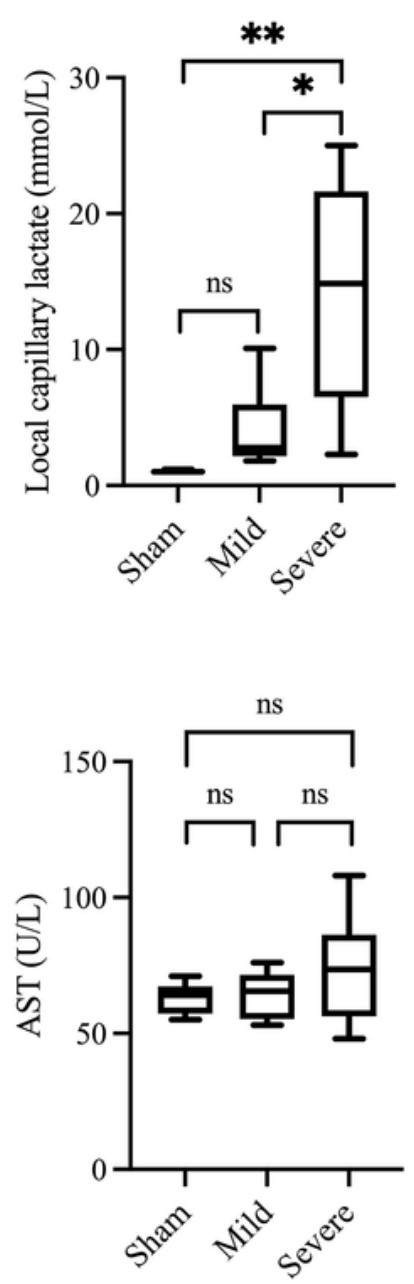
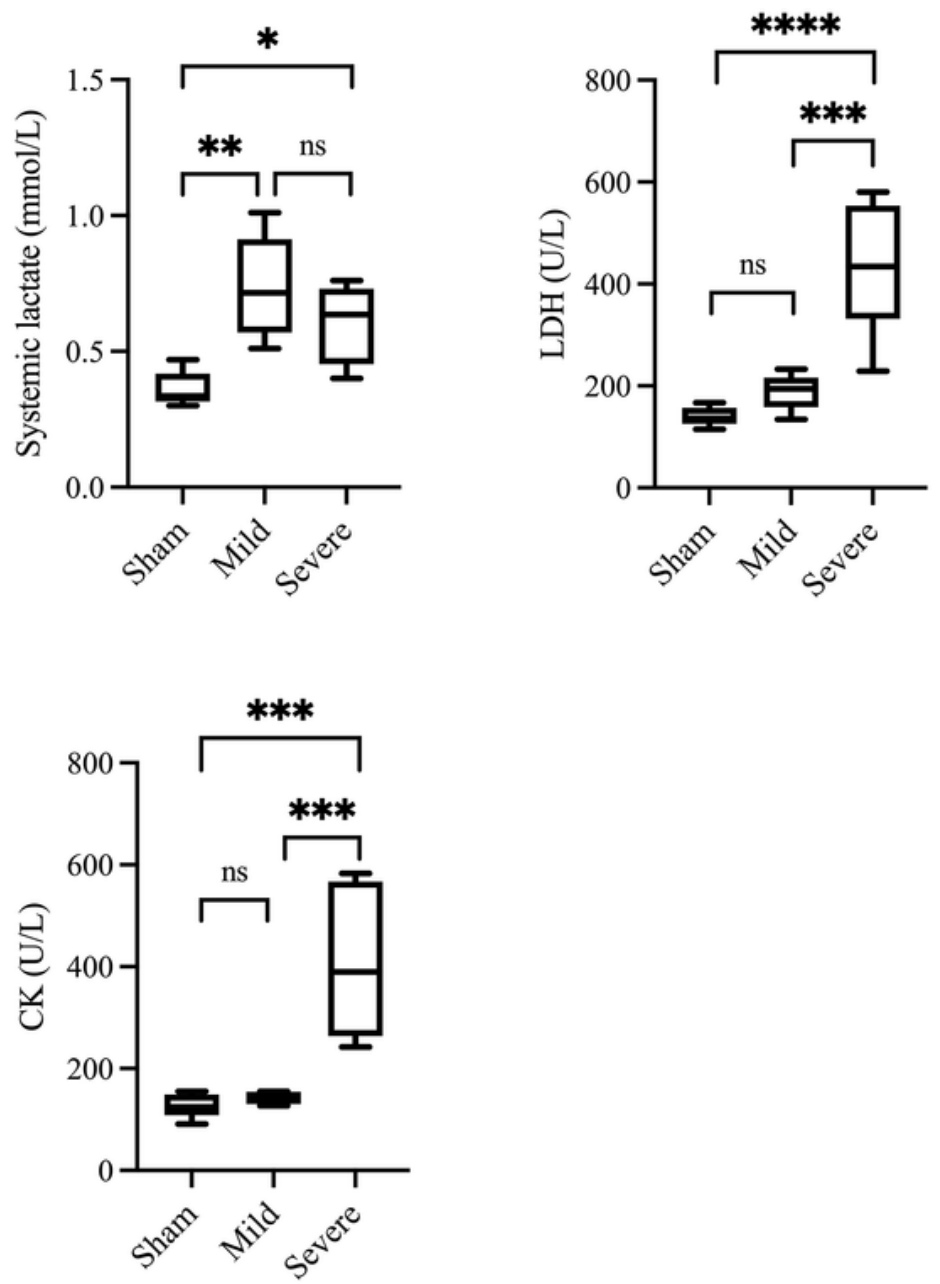

B)

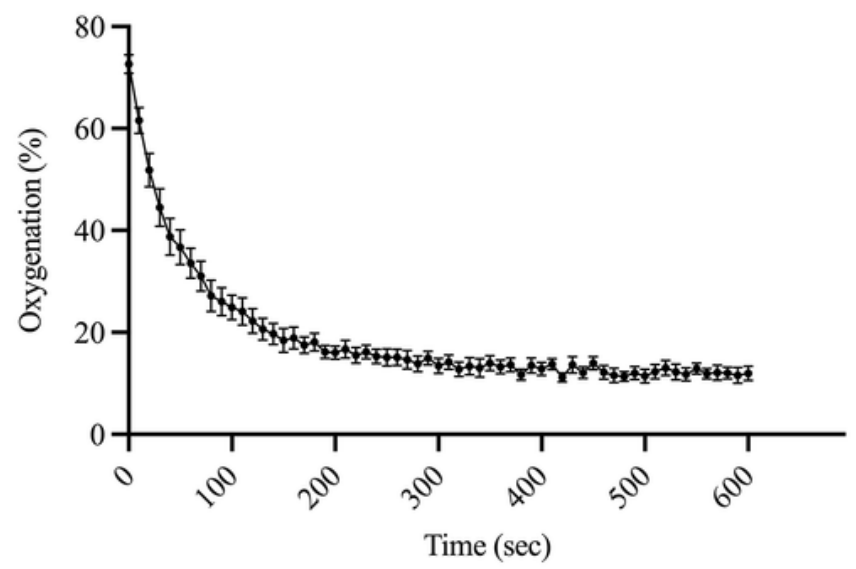

\section{Figure 1}

(A) The results of laboratory tests in the three groups ( $n=6$ rats per group) (B) Time course of mean oxygenation in the early stage of AMI A rapid decrease of oxygenation from baseline (> 70\%) after clamping is clearly visualized. The oxygenation reaches a plateau within 5 minutes. Error bars represent standard errors of the mean ( $n=12$ rats). Note - Sham, sham group; Mild, mild acute mesenteric ischemia group; Severe, severe acute mes-enteric ischemia group; LDH, lactate dehydrogenase; AST, 
aspartate transaminase; CK, creatine phosphoki-nase; ns, not significant; *, $\mathrm{p}<.05 ; * \star, p<.005 ; \star \star \star, p<$ $.0005 ; * \star \star \star, \mathrm{p}<.0001 ; \mathrm{AMI}$, acute mesenteric is-chemia

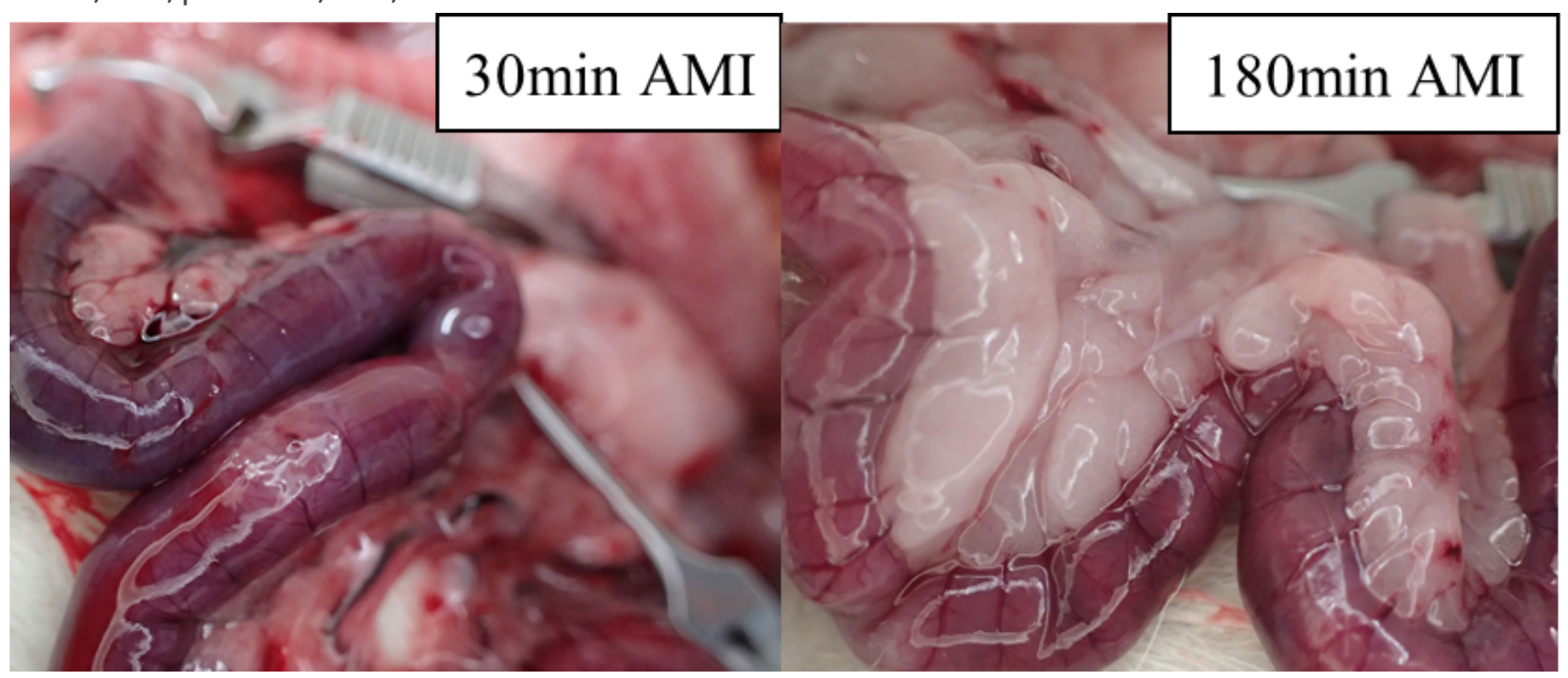

\section{Figure 2}

Gross appearance of ischemic ileum in the mild and severe groups In some cases, the severity of AMI is indistinguishable by the gross appearance alone. Note - AMI, acute mesenteric ischemia 

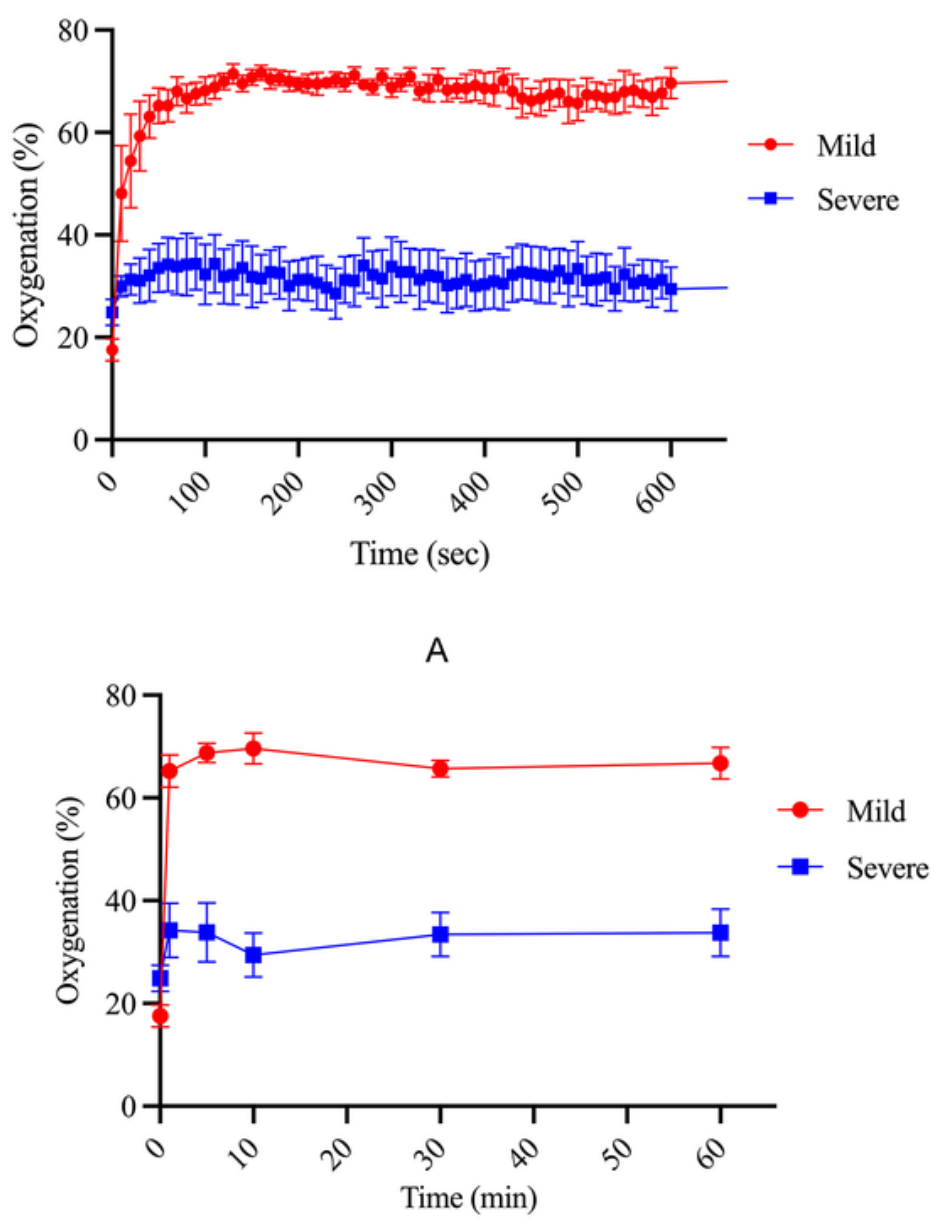

B

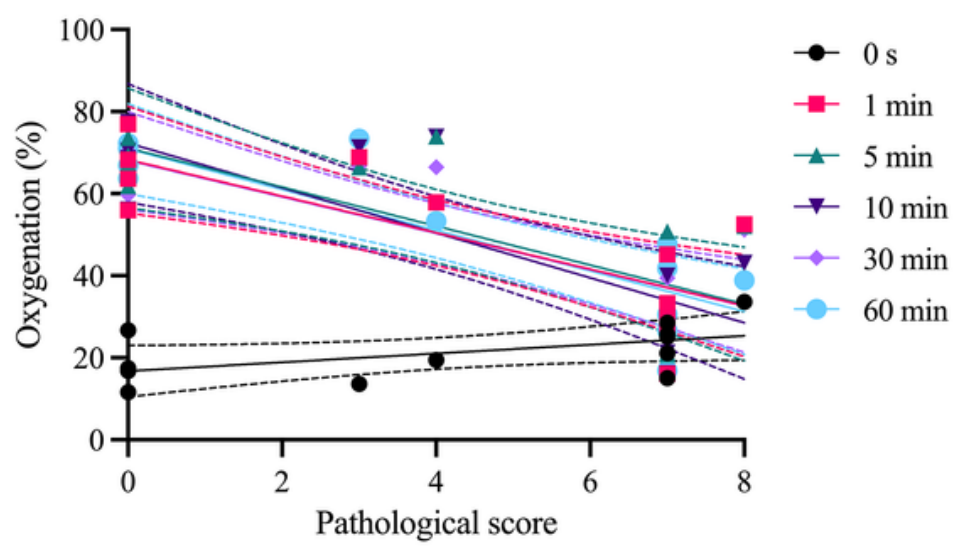

C

\section{Figure 3}

(A) Time course of oxygenation until 10 minutes after reperfusion The mild AMI group shows rapid recovery of oxygenation to the baseline within five minutes, whereas the severe AMI group shows insufficient recovery. Error bars represent standard errors of the mean ( $n=6$ rats per group). (B) Time course of oxygenation until 60 minutes after reperfusion (C) The correlation between oxygenation and the pathological score with the best-fit line with $95 \%$ confi-dence band After reperfusion, there is a negative 
linear relationship between oxygenation and the pathological score. The correlation coefficient (rs) and p values are shown. Note - AMI, acute mesenteric ischemia; Mild, the mild AMI group; Severe, the severe AMl group

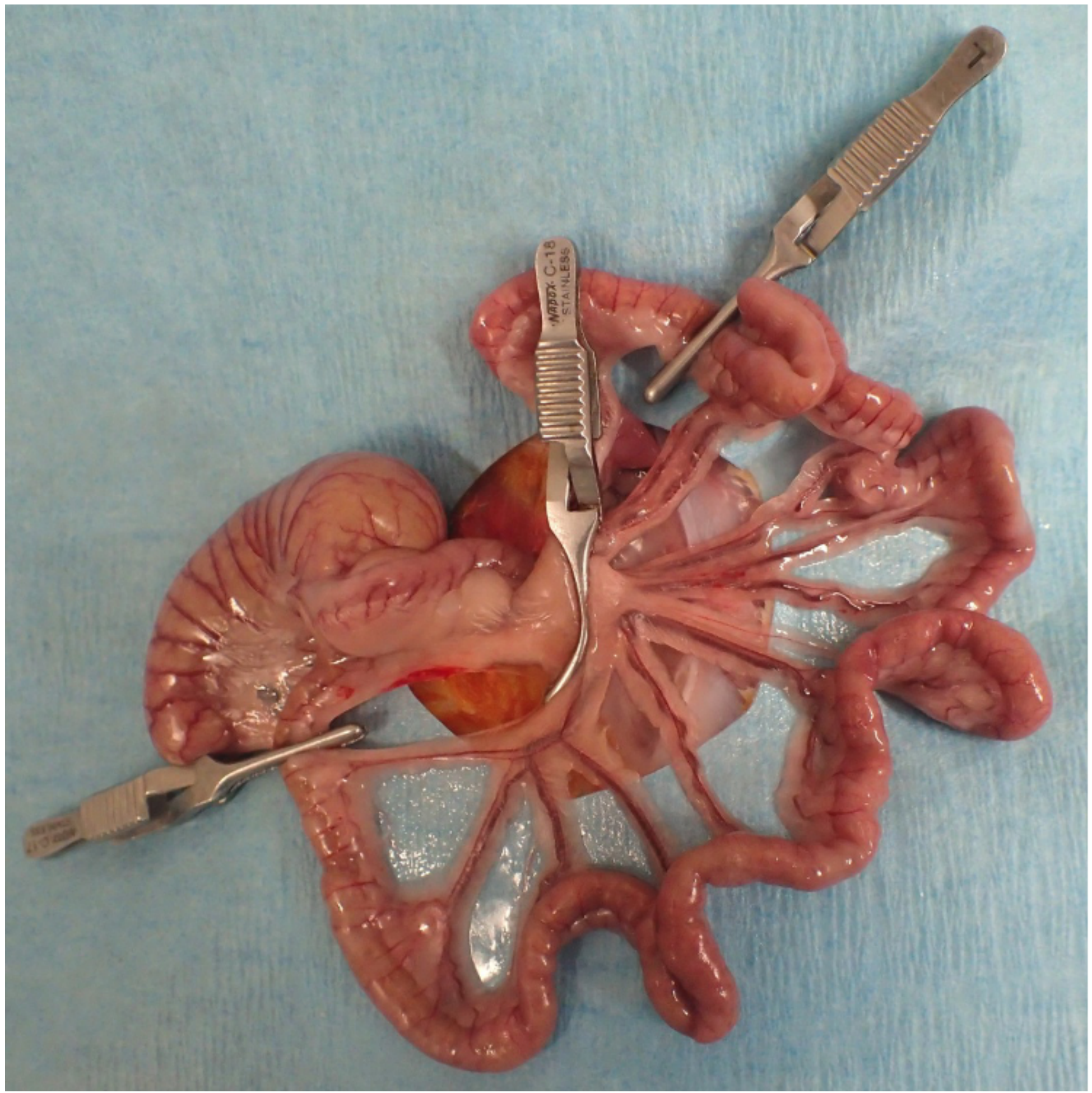

\section{Figure 4}

Strangulated bowel obstruction model in rats Acute mesenteric ischemia was induced in $15 \mathrm{~cm}$ of ileum from the ileocecal valve using vascular clamps. Mesenteric arteries and veins and marginal arteries and veins were clamped. 


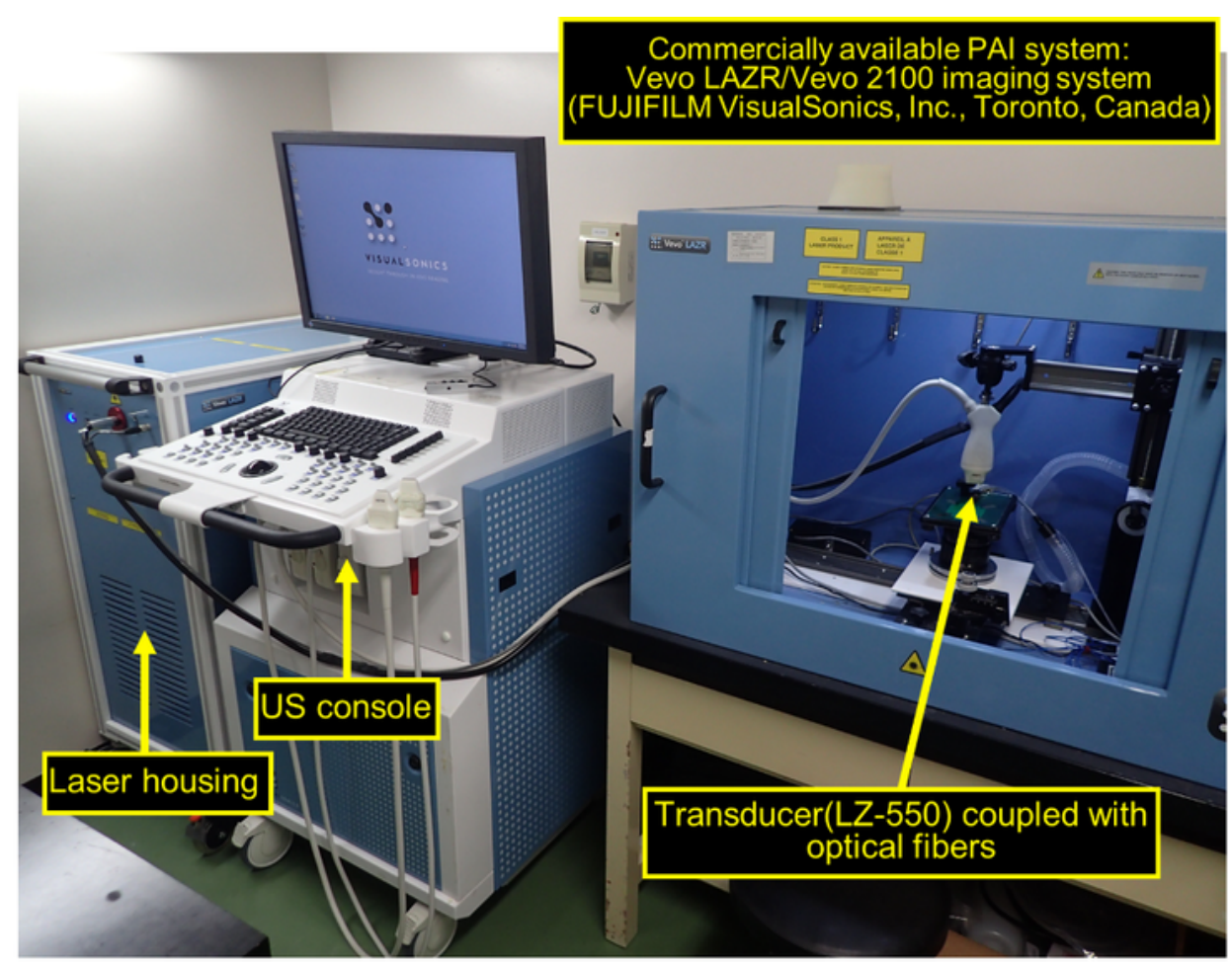

A

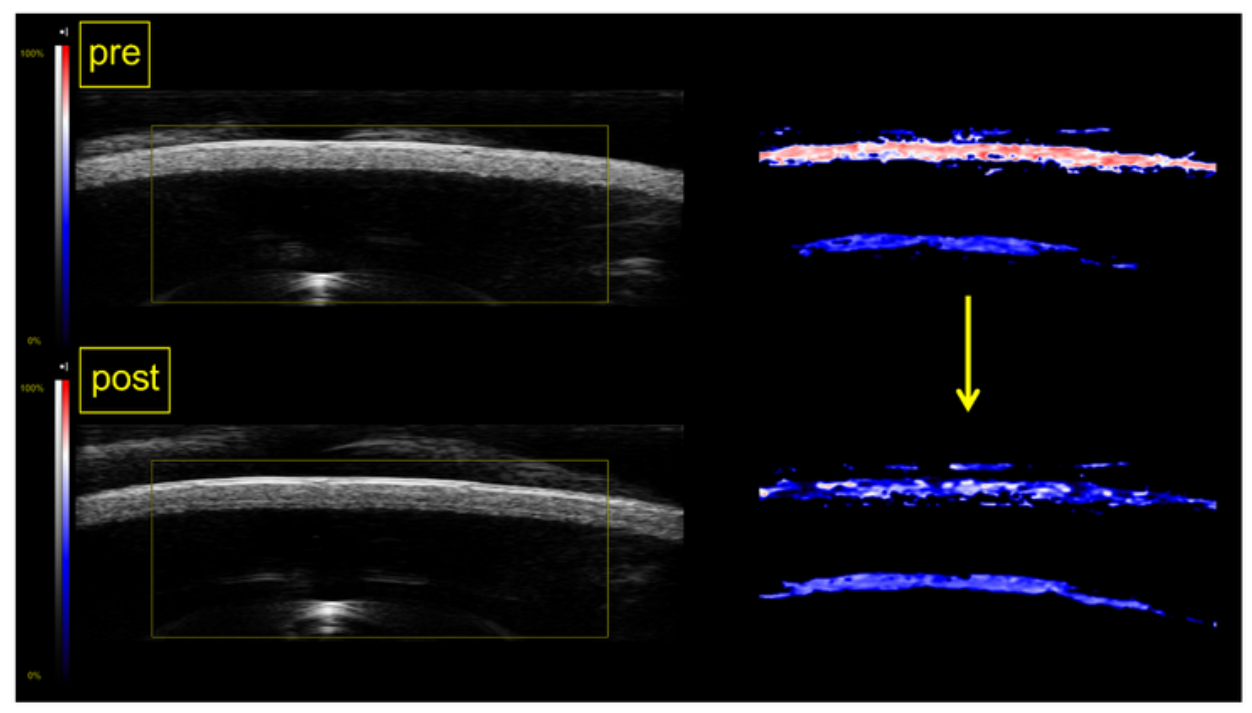

B

\section{Figure 5}

(A) Commercially available photoacoustic imaging systems Vevo LAZR uses handheld transducers for laser delivery and photoacoustic signal detection. (B) Photoacoustic imaging of the distal ileum before and after inducing AMI B-mode image and oxygenation mapping are shown in parallel. Note - AMI, acute mesenteric ischemia 


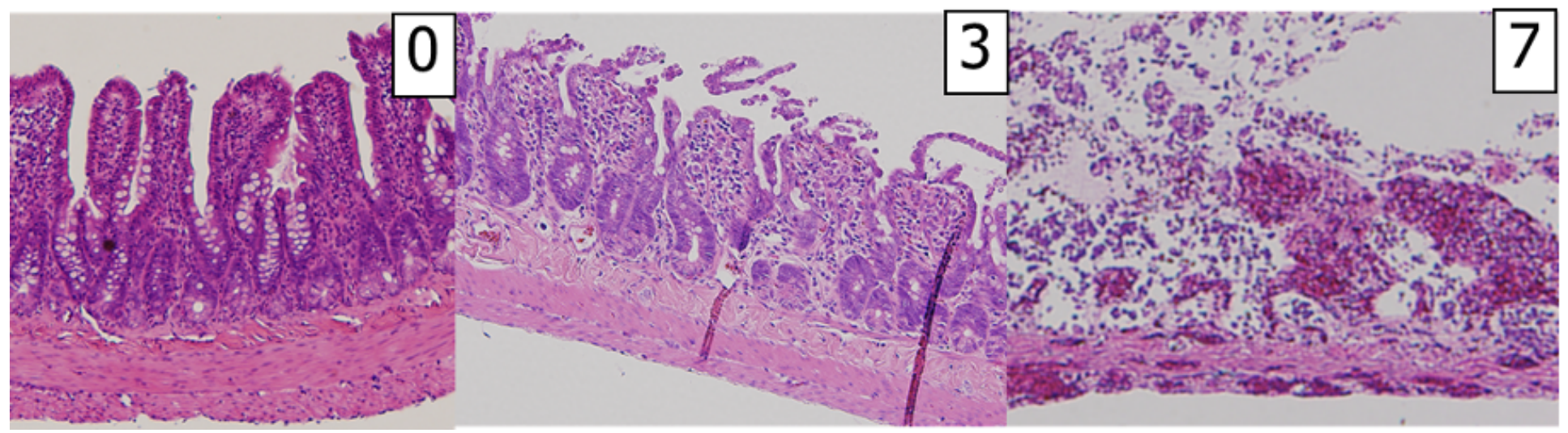

Figure 6

Examples of pathological findings with the pathological scores 0 , normal mucosa; 3 , massive lifting down sides of villi, some denuded tips; 7 , transmucosal infarction. 